Pensamiento Crítico N. ${ }^{\circ} 11$, pp. 65-72

\title{
La inducción embrionaria en Sócrates
}

Víctor Orozco Livia

\section{RESUMEN}

La propuesta de una primera forma ordenada de pensar en torno a la búsqueda de la verdad, es un mérito que le corresponde al filósofo griego Sócrates.

Sócrates representó en su época a la mayor virtud intelectual de los pensadores griegos, surgidos al amparo de la magnificencia de Atenas en el mundo antiguo. Atenas floreció a mediados del siglo $\mathrm{V}$ a.C, después de que se produjeran diversos acontecimientos sociales, políticos e intelectuales importantes en el marco de un régimen esclavista que tendía a acentuar sus características.

Ocurrió la caída del Areópago, que era el centro elitista de la aristocracia conservadora, y se instauró un mayor espacio democrático para los ciudadanos, el triunfo militar de Grecia contra los persas expandió el intercambio comercial y cultural de Atenas con las ciudades más importantes de la época, y la controversia de Sócrates con los sofistas respecto a la verdad, etc., fueron algunos de los factores de una convergencia que influyeron en las ideas de Sócrates. En medio de tales acontecimientos Sócrates propuso un inicial método de inducción.

Palabras claves: Inducción, embrionaria, Sócrates, sofistas, mayéutica.

\section{ABTRACT}

The first proposal for an orderly way of thinking about seeking the truth, is a merit that belongs to the greek philosopher Socrates. 


\section{Víctor Eduardo Orozco Livia}

Socrates in his time represents the highest intellectual virtue of greek thinkers, arising under the magnificence of Athens in the middle of $V$ century bc, wich occurred after several social events, politicians and intellectuals in the context of a slave regimen that tended to accentuate their features.

It happened that the fal of the Areopagus who was the center of the elitist and conservative aristocracy opened a more democratic space for citizens, the military victory of Greece against the Persians expand trade and cultural exchanges by Athens with the most important cities of the time, and dispute with the sophists by Socrates about the truth, and so on, were some of the converting factors that influenced the ideas of Socrates.

Amid such delopments Socrates proposed an initial induction method.

Keywords: Induction, embryonic, Socrates, sophists, mayéutica.

\section{INTRODUCCIÓN}

La inducción cumple un rol sumamente importante en las investigaciones científicas hoy en día. La aparición de la inducción y su posterior desarrollo obedeció a vicisitudes de diverso orden que se dieron de acuerdo a los requerimientos de cada época.

Sócrates puso los cimientos de la inducción en una época de contexto social convulsionado y en frontal controversia con los filósofos autodenominados sofistas.

En gran parte de la época medieval la inducción no hizo mayores progresos y más bien se prefirió el razonamiento deductivo propuesto por Aristóteles.

Cuando se produce el Renacimiento (Siglo XVI) y se llevan a cabo ensayos experimentales que impulsan el desarrollo de las ciencias naturales reaparece la inducción como abstracción de los procedimientos experimentales. Esta nueva modalidad de razonar polarizó a los filósofos, los que propugnaban la deducción y los que preferían la inducción.

Hubo de aparecer J. S. Mill (1806-1873) para dilucidar este asunto, señalando que primero se hace inducción y solo después se puede hacer deducción, es decir, son se- 


\section{La inducción embrionaria en Sócrates}

cuenciales e indesligados Posteriormente, la filosofía hizo abstracción de otros métodos racionales los que se emplean para hacer ciencia, provistos de determinados principios o valores que anidan en el pensamiento de los investigadores.

\section{EL ROL DE LA INDUCCIÓN EN LA ACTUALIDAD}

Después de un recorrido histórico que abarca aproximadamente 2500 años, la filosofía ha logrado abstracción generalizada de diversas formas de razonar, aplicables a la ciencia y a la filosofía. Algunos de estos métodos racionales son la inducción y la deducción, el análisis y la síntesis, la comparación y la analogía, el raciocinio de teoría de conjuntos, el raciocinio de teoría de juegos, el raciocinio cibernético, el raciocinio sistémico, etc., son métodos de razonamiento que se emplean de acuerdo al tipo de investigación y bajo determinados criterios de valor que conllevan los investigadores. Así también lo considera G. Myrdal, Premio Nobel de Economía en 1975, cuando dice que "la investigación debiera comenzar dando atención a algunas premisas de valor que parecieran apropiadas para utilizarse, pero que deben estar preparadas para ajustarse continuamente ${ }^{1}$. Esto es, valores que la ciencia y la filosofía van captando debido a las abstracciones que hacen sobre una realidad social en movimiento y cambio, de acuerdo a las condiciones imperantes.

Son los valores, básicamente, y no los instrumentos racionales los que direccionan las investigaciones a unas conclusiones u otras. Es la explicación de que en una misma rama científica, circundan varios paradigmas o teorías, reclamando cada una de ellas ser reconocida como científicas en el ámbito académico. Tenemos por ello en el mundo académico actual de las ciencias económicas: la teoría neoliberal, la teoría cepaliana, la teoría institucionalista, la teoría marxista, el resurgimiento de la teoría keynesiana, etc., que pretenden la prioridad de aceptación científica. Como uno de esos raciocinios importantes en el proceso de investigación, la inducción indesligada de la deducción, cumple un destacado papel, no solo en las ciencias naturales y sociales, sino también en las ciencias que estudian las diversas formas del pensamiento.

En la ciencia económica, que es por excelencia una ciencia social, la investigación se inicia con una descripción del fenómeno estudiado, recurriendo para ello a la estadística inferencial. Con tal técnica se obtiene información, se procesa y se presenta

1 Myrdal, G. Objetividad de la Investigación Social. Ed. FCE. México, 1976. Pág. 68. 


\section{Víctor Eduardo Orozco Livia}

la información. La técnica de la estadística inferencial es una derivación del método inductivo. Y en un segundo momento, cuando el tipo de investigación lo requiere se emplea la inducción para hallar relaciones de causa a efecto en el fenómeno investigado. Esta inducción denominada causal adopta diversas modalidades como el método de la concordancia, él método de la diferencia única, el método de la doble concordancia, el método de las variaciones concomitantes y el método de los residuos.

Quien puso los cimientos de esta importante forma de razonar fue el insigne filósofo griego Sócrates, y tiene por ello reservado un especial lugar en el desarrollo de la filosofía, el método y la investigación.

\section{EL CONTEXTO HISTÓRICO EN LA ÉPOCA DE SÓCRATES}

Sócrates nació en Atenas y vivió entre 427 y 347 a. C. No dejó escrito alguno. Se sabe de él por las referencias de sus discípulos como Jenofonte y Platón y por lo que expresó de él Aristóteles, quien cultivó su amistad por cerca de 20 años, en el transcurso de su existencia.

Sócrates presenció el florecimiento de Atenas y su caída, esto último después de la Guerra del Peleponeso. Atenas prosperó después de las Guerras Médicas en las primeras décadas del siglo $\mathrm{V}$ a. C. Después de este triunfo sobre Persia, Atenas se convirtió en el centro del desarrollo de Europa y Asia. Su expansión comercial cubrió las rutas del Mar Mediterráneo, Mar Egeo y el Mar Negro y llegó hasta la ciudad de Mileto en Asia Menor. El intercambio con muchas ciudades era no solo de productos, sino también de carácter cultural.

En el 462 a. C. aconteció que Efialtes al frente de demócratas radicales derrocó al Aeropago, que era la institución que había concentrado por mucho tiempo el poder aristocrático y conservador en Atenas. Tal ruptura permitió el acceso a ciudadanos no necesariamente de la elite aristocrática a participar del gobierno y de las decisiones del Estado, por elección directa y no mediatizada.

Tales acontecimientos dieron lugar a que Sócrates, en su juventud, presenciara cómo en ese nuevo terreno democrático Pericles efectuaba grandes reformas políticas, económicas y culturales. Atenas convertida en la metrópoli cultural y política de Grecia 


\section{La inducción embrionaria en Sócrates}

se convirtió en un centro de ebullición de ideas. Fueron momentos en que Sócrates escuchó a los grandes sofistas como Pitágoras, Gorgias, Prodicó, Hipias, etc., leyó asimismo el famoso tratado sobre la naturaleza de Anaxágoras, asistió a famosas representaciones teatrales trágicas y fue testigo de encendidos debates de gran oratoria.

Tales acontecimientos y su preparación filosófica, que en aquel tiempo englobaba todos los conocimientos de la época, impregnaron el pensamiento de Sócrates y acometió con ellos diversas tareas. Buscar no solo el camino del raciocinio hacia la verdad, enfrentándose a los sofistas, sino también encontrar bases racionales en las que pudieran asentarse los valores imperantes en aquella época. Preocupaciones surgidas a raíz del frustrado proyecto expansionista de Pericles y que culminó con el arrasamiento de Atenas por Esparta. Desde entonces, aquellos valores que propugnaban la guerra, la lucha por el poder, la expansión militar, etc., sin importar los medios y las consecuencias de tales actos, fueron puestos en cuestión, no solo por Sócrates, sino también por historiadores de la época como Tucídides que se quejaba de que "Las garantías de fidelidad recíproca, no las avalaba la Ley divina, sino más bien la complicidad en el crimen" 2 . Implícitamente para los griegos la guerra era necesaria para capturar prisioneros y convertirlos en esclavos. El trabajo esclavizado era la fuente de sus riquezas e inspiraban tales valores, profesadas, por casi todas las naciones de esa época.

Y por pretender encontrar justificaciones racionales a los valores políticos y religiosos, que se basaban en disposiciones puramente teleológicas, fue acusado Sócrates por el Estado ateniense de corromper a la juventud y de introducir nuevos dioses. Juzgado y condenado a muerte, le propusieron como última gracia el destierro pero el filósofo de la inducción en ciernes, respondió que lo mismo le ocurriría en cualquier otro Estado por sus ideas, prefirió beber la cicuta. Con su muerte quedó postergado por mucho tiempo el proyecto de razonar con inducción.

\section{DE LA SOFÍSTICA A LA MAYÉUTICA}

En pleno florecimiento de Atenas, siglo $\mathrm{V}$ a. C., apareció una corriente de pensadores que se autoproclamaban como sabios, en griego sofhistes, y que alcanzaron cierta notoriedad en la población griega. A diferencia de la filosofía presocrática que le interesaban los problemas de la naturaleza, los sofistas priorizaron los problemas de

2 Tucídides "Historia de la guerra del Peloponeso". Ed. Guadarrama. Madrid, 1976. Pág. 105. 


\section{Víctor Eduardo Orozco Livia}

los seres humanos y empezaron a difundir conocimientos cobrando por sus servicios. Mayormente apuntaban a la elite aristocrática para preparar a sus miembros en asuntos públicos y políticos. Los sofistas se preocupaban por la técnica del dominio de la retórica en los discursos. Solían decir los sofistas que nadie está en condiciones de probar que las explicaciones sobre la naturaleza, sobre los dioses, sobre las virtudes morales y las costumbres, son verdaderas. Por consiguiente, la verdad para los sofistas dependía de quien pudiera imponer sus criterios con destreza en la oratoria. Según el filósofo sofista griego Gorgias (483-375 a.C.) "la palabra es poderoso soberano que consigue los más maravillosos efectos con el órgano más pequeño e insignificante. Pues, consigue espantar el miedo, desterrar al dolor, suscitar la alegría y despertar compasión"3. Era la búsqueda de un fin sin raciocinio. A Sócrates no le convence la formalidad discursiva y trata de buscar el camino racional a la verdad. Es consciente de esta deficiencia, y por ello Sócrates no se presentaba como sabio o maestro, sino como filósofo, esto es, el que tiene "amor al saber", y por consiguiente a diferencia de los sofistas decía que no se dedicaba a enseñar sino a aprender de los demás. Su réplica a los sofistas que se autoproclamaban como sabios se plasmaba en su famosa sentencia "solo sé que nada sé", poniendo a la orden del día la necesidad de encontrar el camino racional hacia la verdad o a las definiciones universales, como solía decir. Fiel a sus principios, Sócrates recorre calles y plazas públicas, propiciando el diálogo que conduzca a las definiciones. Son diálogos dirigidos por Sócrates en torno a un tema y cuyo procedimiento consta de dos momentos: la ironía y la mayéutica.

En un primer momento, Sócrates propicia el intercambio de opiniones sobre un tema determinado, el diálogo se inicia con preguntas que formula Sócrates a su interlocutor. Ante las respuestas, Sócrates se coloca en una posición de crítico mostrando las deficiencias de tales respuestas. Es la fase que se ha dado en llamar la ironía.

En un segundo momento, Sócrates lleva a que el propio interrogado se esfuerce en dar respuestas o definiciones sobre el tema, más apropiadas o cercanas a la verdad. Es el momento de la mayéutica que como decía el propio Sócrates, era una labor que cumplía en forma muy parecida a la que hacía su madre, de profesión comadrona, ayudar a dar a luz, en este caso ayudar a dar a luz la verdad. Era por cierto una inducción muy precaria aún, opinática, estrictamente cualitativa, no causal y sin remisión de prueba a los hechos, etc., pero para la época constituyó un paso cualitativo gigantesco en el ordenamiento

3 Citado por F. Muñoz y F. Bobbio en Filosofía de la educación. UNMSM. Lima, 2000. Pág. 86. 


\section{La inducción embrionaria en Sócrates}

del pensar para arribar a una conclusión. Por ello, el filósofo García Morente no tiene ningún reparo en reconocer que, "Sócrates es en realidad el primer filósofo que nos habla de su método"4 el de la mayéutica, a que se refiere Sócrates.

La esencia de la ciencia y la filosofía es el razonamiento ordenado para arribar a una conclusión. La ciencia razona sobre aspectos particulares de la realidad y la filosofía sobre el ser, y por eso Sócrates que dio el primer paso en pensar ordenadamente, tiene el mayor reconocimiento de la intelectualidad mundial.

\section{BIBLIOGRAFÍA}

Cornforth, M. Ciencia versus idealismo. Ed. Lautaro. Buenos Aires, 1955.

Korshunov A. M. Actividad, conciencia y conocimiento científico. Ed. Nueva Cultura. Lima, 2006.

Marías, J. Historia de la filosofía. Ed. Alianza Universidad. Madrid, 1985.

Navarro M. y Calvo T. Historia de la filosofía. Ed. Anaya. Madrid, 1990.

Sisco A. y Eguilior N. Historia 1. Ed. Kapelusz. Buenos Aires, 1981.

4 García Morente "Lecciones Preliminares de Filosofía”. Ed. Diana, México, 1964, pág. 20. 